\title{
A 3-year Follow-up Study on the Alternating Use of Static Splints after Metacarpophalangeal Joint Arthroplasty in a Patient with Rheumatoid Arthritis
}

\author{
Hiroyuki Hayashi, BS, OTR¹, Jun Uchiya, OTR² \\ ${ }^{1}$ Division of Occupational Therapy, Faculty of Care and Rehabilitation, Seijoh University \\ 2 Rehabilitation Division, Gifu University Hospital
}

\begin{abstract}
Burr et al. (2002) reported the results of a 19-month follow-up study on a static splinting regimen as an alternative to dynamic extension splinting after metacarpophalangeal (MCP) joint arthroplasty. However, the long-term results of static splinting therapy have not been reported. Therefore, we conducted a 3-year follow-up study on the use of alternating static splinting in extension and flexion after MCP joint arthroplasty. Active flexion and extension of the MCP joints and grip strength were evaluated before surgery and at 12 weeks and 3 years after surgery. The range of motion (ROM) of the MCP joints and grip strength improved at 12 weeks and 3 years postoperatively. The efficacy of dynamic extension splinting after MCP joint arthroplasty indicated no change in the ROM and grip strength. The findings in this case indicate the positive long-term results of a static splinting regimen.
\end{abstract}

Key words: rheumatoid arthritis, splint, range of motion

(Asian J Occup Ther 9: 1-5, 2011)

\section{Introduction}

Common hand deformity in rheumatoid arthritis (RA) results from the destruction and dislocation of the metacarpophalangeal (MCP) joints and ulnar deviation of the finger (Chung, Kotsis \& Kim, 2004).

Hand deformities caused by RA are often treated with surgery and rehabilitation to improve a patient's hand function-associated activities of daily living (ADLs) (Mannerfelt \& Andersson, 1975; O'Brien, Jones, Mullis, Mulherin \& Dziedzic, 2006).

MCP joint arthroplasty with implants has been adopted for treatment of RA patients since the 1960s (Swanson, 1972), because RA can damage the MCP joints resulting in flexion and ulnar deviation deformities. Surgical intervention via MCP joint arthroplasty using Swanson implants has been performed for improving hand function.

The use of a dynamic extension splint has been rec-

Received: 5 March 2010, Accepted: 23 February 2011

Corresponding to: Hiroyuki Hayashi; Division of Occupational

Therapy, Faculty of Care and Rehabilitation, Seijoh University, 2-172

Fukinodai, Tokai, Aichi 476-8588, Japan

Phone: 81-52-601-6451 Fax: 81-52-601-6451

e-mail: hayashi-h@seijoh-u.ac.jp

(C2011 Japanese Association of Occupational Therapists ommended after MCP joint arthroplasty (Swanson, 1972). A number of studies have reported that this method results in an increased extension MCP joint range of motion (ROM), but a decreased flexion (ROM), especially in the ulnar digits (Rothwell, Cragg \& O’Neill, 1997; Moller, Sollerman, Geijer, Kopyylov \& Tagil, 2005). Furthermore, Blair, Shurr \& Buckwalter (1984), Bieber, Weiland \& Volenec-Dowling (1986), and Delaney, Trail \& Nuttall (2005) indicated that there were no changes in pre- or postoperative grip strength.

We have been carrying out dynamic splinting as therapy for patients undergoing MCP joint arthroplasty, and although MCP joint extension can be improved, our experience shows that MCP flexion and grip strength decrease after surgery. This finding is consistent with those of previous reports (Blair et al. 1984). Restricted MCP joint flexion and decreased grip strength may lead to increased difficulties in performing ADLs such as gripping a knife to cut food or squeezing a towel.

On the other hand, Burr, Pratt \& Smith (2002) suggested the usefulness of alternating daily the application of static extension and flexion splints. Their study reported improved extension and flexion ROM. Furthermore, as compared to the results of the method recommended by Swanson (1972), the flexion ROM was much improved. 
Table 1. Postoperative MCP joint ROM with dynamic extension splinting

\begin{tabular}{|c|c|c|c|c|c|c|c|c|}
\hline & \multicolumn{4}{|c|}{ Extension (degrees) } & \multicolumn{4}{|c|}{ Flexion (degrees) } \\
\hline & Index & Long & Ring & Small & Index & Long & Ring & Small \\
\hline Our result & 26 & 20 & 16 & 26 & 60 & 56 & 46 & 52 \\
\hline Mannerfelt et al. & 8 & 12 & 10 & 4 & 51 & 55 & 50 & 39 \\
\hline Goldfarb et al. & 23 & 27 & 24 & 16 & 60 & 60 & 60 & 49 \\
\hline
\end{tabular}

Their study also highlighted the comfort, simple design, ease of production, and low cost as added advantages of the alternating splinting method. The purpose of this study was to investigate the 3-year follow-up results of alternating static splinting after MCP joint arthroplasty.

We adopted the alternating static splinting method after MCP joint arthroplasty rather than the dynamic splint because we believe that it is easier to both manipulate the splints after being discharged and flex the MCP joints while wearing the splints.

\section{Case}

The patient was a woman in her sixties whose condition had been diagnosed as RA 14 years previously. The patient had functional motion of the shoulder, elbow and wrist. However, because of the ulnar deviation and subluxation of the fingers at the MCP joints, the patient experienced weak palmar grip. Therefore, she was surgically treated. First, Swanson implants were inserted in $4 \mathrm{MCP}$ joints of her right hand to correct the ulnar drift, and the palmar subluxation as well as to relieve pain. After the MCP joint arthroplasty, dynamic splinting was performed. At the $12^{\text {th }}$ postoperative week, the results were nearly the same as those reported in other studies (Mannerfelt \& Andersson, 1975; Goldfarb \& Stern, 2003) (Table 1).

Nine months after the initial MCP joint arthroplasty, bridging calluses developed due to bone spurs near the MCP joints of the ulnar digits. Approximately 1 year after the initial MCP joint arthtroplasty, the MCP joint ROM of the ulnar digits was diminished. Therefore, the bone spurs were surgically removed and the implants in all the MCP joints were replaced. After the second MCP joint arthroplasty, we employed an alternating static splinting method.

The patient was prescribed methotrexate (MTX) and prednisolone in the case of the dynamic extension splinting. However, tacrolimus hydrate and prednisolone were prescribed in the case of the alternating static splinting.

\section{Progress and Rehabilitation Program}

\section{Evaluation}

Although the postoperative rehabilitation program was completed 12 weeks after the second operation, a fol-

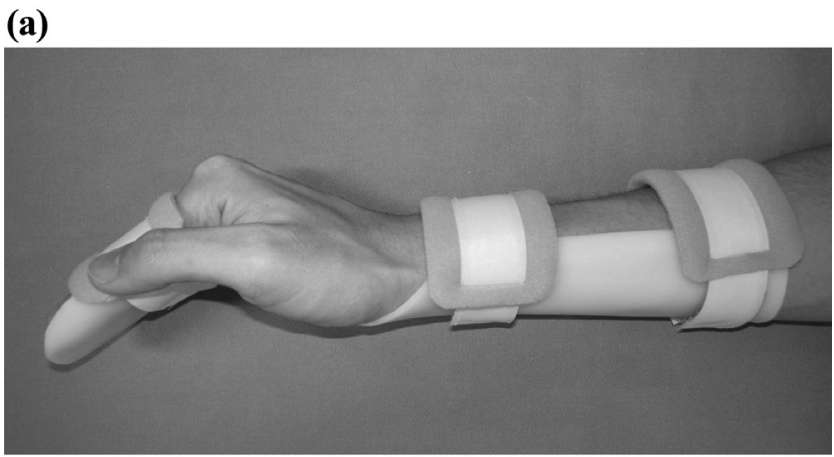

(b)

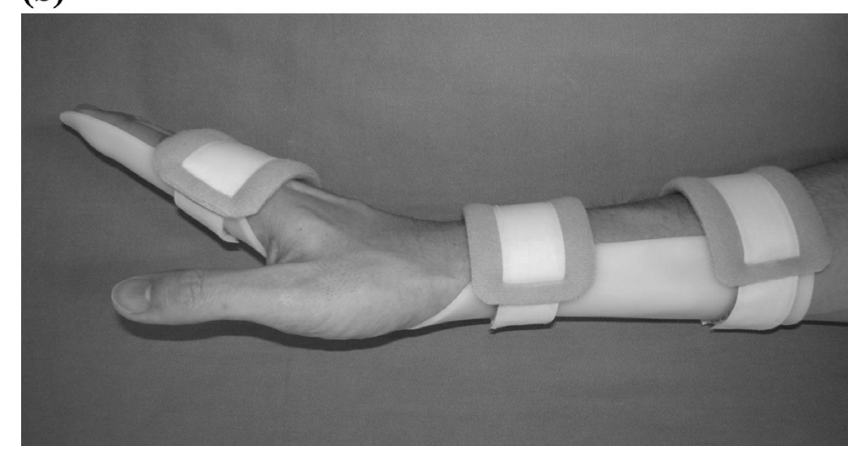

Fig. 1. Alternate splints of same type with splints the patient wore. The wrist is extended at 30 degrees, the MP joints are flexed at 70 degrees, and IP joints are extended at 0 degrees (a). The wrist is extended at 30 degrees, and the MP and IP joints are extended at 0 degrees (b).

low-up rehabilitation program continued for 3 years. Objective measurements of active ROM of the MCP joints in flexion and extension were obtained with a small goniometer over the dorsum of the digital joints. The arc of active motion was determined by subtracting the active extension lag value from the active flexion value. Grip strength was measured using the JAMAR hand dynamometer.

Active flexion and extension of the MCP joints and grip strength were evaluated preoperatively (before the first and second operations) and at 12 weeks and 3 years after the second operation.

\section{Alternating static splinting}

Exercise and splinting were conducted according to the rehabilitation plan reported by Burr et al. (2002). Rehabilitation began on the third postoperative day. Static 
Table 2. Rehabilitation after MCP joint arthroplasty

\begin{tabular}{ll}
\hline Time Frame & \multicolumn{1}{c}{ Therapeutic Intervention } \\
\hline 3-4 wk postop & $\begin{array}{l}\text { Active ROM initiated for MCP and IP joints every hour } \\
\text { 2 splints used alternatively for 24 hours at a time } \\
\text { Operated hand not used for ADLs at first 4 weeks }\end{array}$ \\
& $\begin{array}{l}\text { Active ROM was continued } \\
\text { 4-8 wk postop }\end{array}$ \\
& $\begin{array}{l}\text { Patient began to perform light activities at 4 postoperative weeks } \\
\text { 8-12 wk postop }\end{array}$ \\
& Active ROM was continued until 12 postoperative weeks \\
& 2 splints were worn alternatively only at night until 12 postoperative weeks \\
& The patient began using the operated hand for normal ADLs \\
\hline
\end{tabular}

extension and flexion splints were used for 12 weeks (Fig. 1).

With the static extension splints the MCP and interphalangeal (IP) joints were extended at 0 degrees. With the static flexion splints the MCP joints were flexed at 70 degrees, and the IP joints were extended at 0 degrees. With both splints, the wrist was extended at 30 degrees (Fig.1). The basic aim of using the static extension splint was to maintain the MCP joints at maximum extension, whereas with the static flexion splint we aimed to achieve maximum flexion by extending the collateral ligament and articular capsule, and by excursing the extensor digitorum tendon distally.

The 2 splints were worn on alternate days for 24 hours at a time.

\section{Postoperative rehabilitation program}

The therapeutic intervention provided to the patient is shown in Table 2.

During the day, 2 types of exercises were performed. The MCP and IP joints together were actively extended and flexed, after which the MCP joints were actively flexed and extended; the IP joints were completely extended throughout. This sequence was performed 10 times every hour for 12 weeks after surgery.

The patient was advised to remove the splints every hour during the exercise during the first 4 postoperative weeks; moreover, she was instructed not to use the affected hand during these 4 weeks. After 4 postoperative weeks, the patient was allowed to use the affected hand for light ADLs such as fastening buttons and using a fork. At the $8^{\text {th }}$ postoperative week, normal ADLs such as squeezing a towel and opening a tight jar were permitted. Thereafter, the patient was instructed to alternate between the 2 splints only at night until the $12^{\text {th }}$ postoperative week. Therapy was completed by the end of the $12^{\text {th }}$ postoperative week.

The patient was discharged three weeks after surgery, and the patient subsequently received regular outpatient treatment twice weekly for 3 months.

\section{Results}

Figure 2 shows the ROM and the grip strength of the 2 different splinting methods.

The ROM of the MCP joints before the first operation was $16(-40 / 56)$ degrees in the index finger, 24 $(-20 / 44)$ degrees in the middle finger, $12(0 / 12)$ degrees in the ring finger, and $14(-46 / 60)$ degrees in the little finger. The ROM of the MCP joints before the second operation was $24(-40 / 64)$ degrees in the index finger, $40(-26 / 66)$ degrees in the middle finger, $0(-26 / 26)$ degrees in the ring finger, and $0(-28 / 28)$ degrees in the little finger. The ROM at the $12^{\text {th }}$ postoperative week with the use of alternating splinting was $24(-40 / 64)$ degrees in the index finger, $46(-20 / 66)$ degrees in the middle finger, $48(-16 / 64)$ degrees in the ring finger, and $34(-36 / 70)$ degrees in the little finger. The ROM three years after surgery was 32 $(-40 / 72)$ degrees in the index finger, $48(-22 / 70)$ degrees in the middle finger, $58(0 / 58)$ degrees in the ring finger, and $32(-40 / 72)$ degrees in the little finger (Fig. 3). The passive MCP joint extension at the $12^{\text {th }}$ postoperative week for both operations was 0 degrees.

In addition, grip strengths before the first and second operations were 3.0 and $2.0 \mathrm{~kg}$ respectively. It increased to $6.0 \mathrm{~kg}$ after 12 weeks of therapy using alternating splinting, and 3 years after the second operation it increased to $8.0 \mathrm{~kg}$.

Subjective patient comments indicated a preference for the alternating static splinting therapy. The patient judged dynamic extension splinting to be heavy on the hand and not easy to wear. The patient also found it difficult to flex the fingers. On the other hand, the patient judged the alternating static splinting to be lighter on the hand and easier to wear. The patient also reported increased facility in finger flexion.

\section{Discussion}

In general, dynamic splinting is implemented to maintain and increase the ROM of the MCP joints after MCP joint arthroplasty. Blair et al. (1984) reported that dynamic splinting increased active extension. However, it has been 


\section{Progression of Index MCP motion}

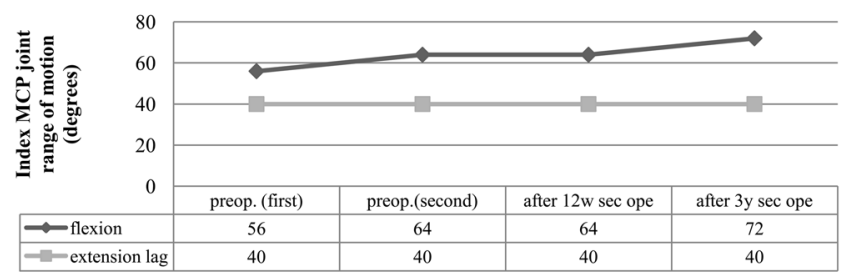

\section{Progression of Middle MCP motion}

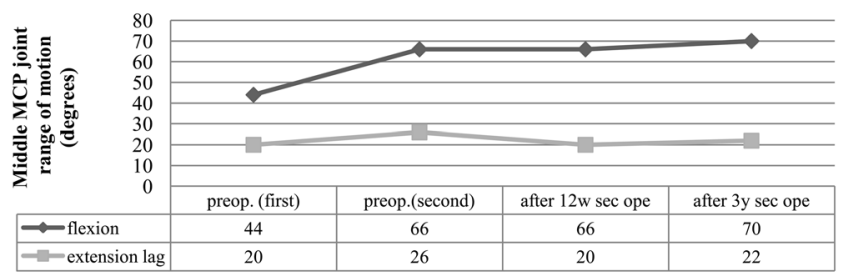

\section{Progression of Ring MCP motion}

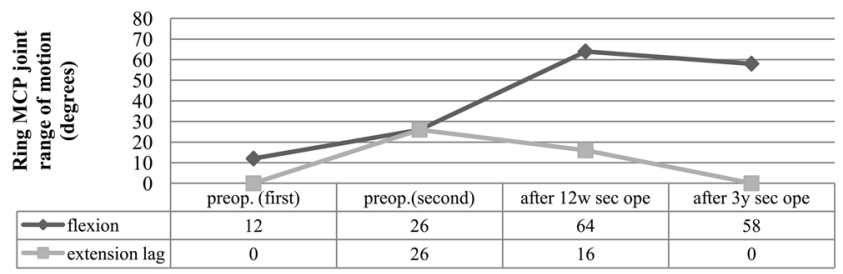

(a)

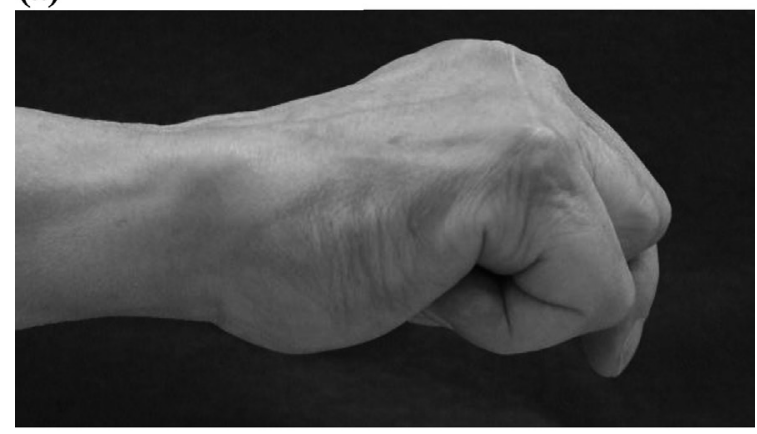

(b)

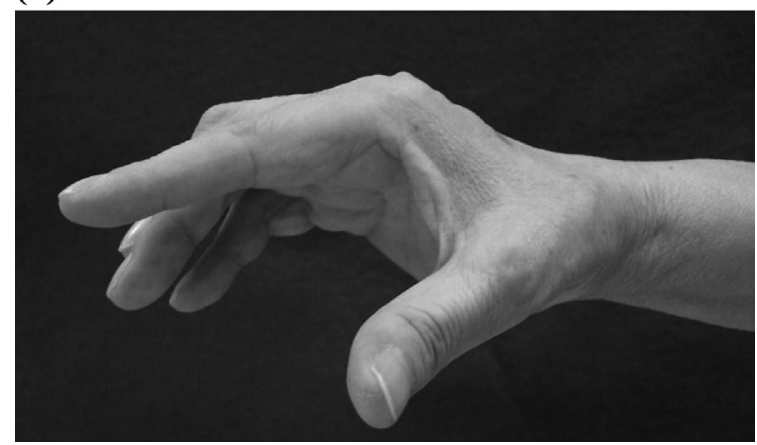

Fig. 3. Flexion (a) and extension (b) of the MCP joints three years after surgery.

\section{Progression of Little MCP motion}

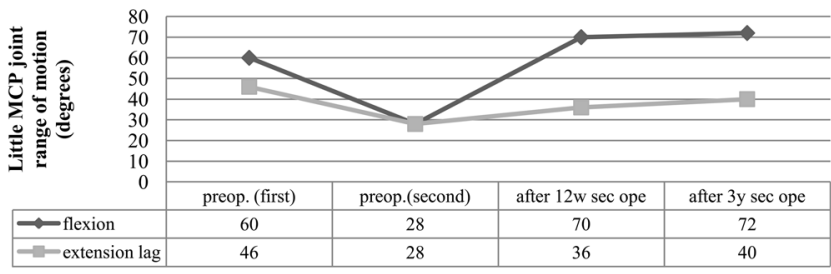

Progression of Grip strength

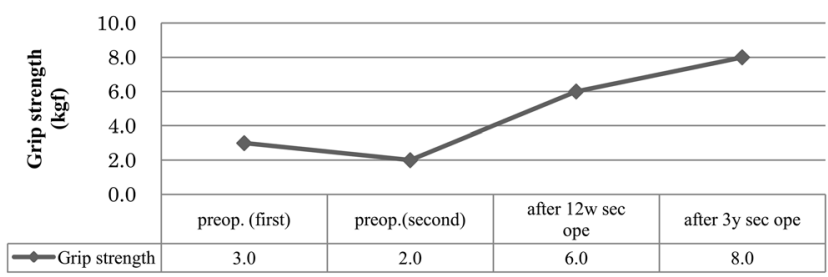

Fig.2. ROM of the MCP joints and grip strength before the first and second operationand at 12 weeks and 3 years after the second operation.

reported that both active flexion of the MCP joints and grip strength using dynamic splinting decreased on average from $72 \mathrm{~N}$ to $62 \mathrm{~N} 54$ months after surgery. O’Brien (1996) stated that there was no significant change in motion. However, our patient's motion and grip strength improved after 3 years as compared with the motion grip strength before the first and second operation, and those at 12 weeks after the second operation. el-Gammal \& Blair (1993) reported that the best motion is during the first 2 years, but the ROM tends to decrease thereafter. However, our patient continued to show improved palmar grip after 3 years.

MCP joints are commonly known to be the most important joints for hand function; they contribute to $77 \%$ of the total arc of finger flexion (Moran \& Berger, 2003) and are important for MCP joint flexion. However, flexing the $\mathrm{MCP}$ joint against the resistant forces of a dynamic splint can be very difficult for some patients (Burr et al., 2002). On the other hand, a static splint placed at MCP joint maintains the MCP joints at 70 degrees in a flexion position. Therefore, it is easy to increase the flexion angle of the MCP joint.

Active flexion ROM of the MCP joints and adequate grip strength is an important pre-requisite for various ADLs such as cutting vegetables with a kitchen knife, turning on the water tap, unscrewing the lid of a jar, or squeezing a towel. Alternating static splinting resulted in higher grip strength as compared to the grip strength 
achieved with dynamic splinting after the first operation, and the grip strength further improved over 3 years.

Hume, Gellman, McKellop \& Brumfield (1990) reported that the average functional ROM of the MCP joints should be 61 degrees to perform ADLs. Therefore, a flexion ROM of more than 60 degrees has to be achieved in the case of the MCP joints. In this patient, with the use of alternating static splinting, the flexion of the MCP joints was maintained at over 70 degrees for 3 postoperative years, except in the case of the ring finger. Thus, a functional ROM of the MCP joints was maintained in this patient. Three years after surgery, the increase in the ROM of the MCP joints as well as the increase in grip strength was due to fact that the patient used the affected hand frequently for ADLs 12 weeks after surgery, thus achieving MCP joint flexion.

The static splint is easy to put on and comfortable to wear; it is also aesthetically pleasing. Furthermore, this splint is not disturbed during ADLs as compared to the dynamic splint. In addition, when getting dressed or walking the outrigger of the dynamic splint sometimes damages clothes, but this does not occur with static splints.

However, the improvement in the extension ROM of the MCP joints was not as significant as that reported by Burr et al. (2002). This may be due to insufficient exercising of the affected hand.

The use of alternating static splints can be useful for the treatment of patients after MCP joint arthroplasty. The ROM of the MCP joints and grip strength achieved in the case of our patient suggests that alternating static splinting could be an effective postoperative therapy.

\section{Conclusion}

We investigated the 3-year progress of a patient with the use of alternating static splints after MCP joint arthroplasty for RA. Three years after surgery, MCP joint flexion and grip strength continued to improve. Therefore, alternating static splinting may be an effective postoperative therapy and could be used as a suitable alternative for dynamic MCP extension splinting.

\section{References}

Bieber, EJ., Weiland, AJ., \& Volenec-Dowling, S. (1986). Silicone-rubber implant arthroplasty of the metacarpophalangeal joints for rheumatoid arthritis. The Journal of bone and joint surgery. American volume. 68, 206-209. [Medline]

Blair, WF., Shurr, DG., \& Buckwalter, JA. (1984). Metacar- pophalangeal joint implant arthroplasty with a Silastic spacer. The Journal of bone and joint surgery. American volume. 66, 365-370. [Medline]

Burr, N., Pratt, AL., \& Smith, PJ. (2002). An alternative splinting and rehabilitation protocol for metacarpophalangeal joint arthroplasty in patients with rheumatoid arthritis. Journal of hand therapy. 15, 41-47. [Medline] [CrossRef]

Chung, KC., Kotsis, SV., \& Kim, HM. (2004). A prospective outcome study of Swanson metacarpophalangeal joint arthroplasty for the rheumatoid hand. The Journal of hand surgery. 29, 646-653. [Medline] [CrossRef]

Delaney, R., Trail, IA., \& Nuttall, D. (2005). A comparative study of outcome between the neuflex and Swanson metacarpophalangeal joint replacements. Journal of hand surgery (Edinburgh, Scotland). 30, 3-7.

el-Gammal, TA., \& Blair, WF. (1993). Motion after metacarpophalangeal joint reconstruction in rheumatoid disease. The Journal of hand surgery. 18, 504-511. [Medline] [CrossRef]

Goldfarb, CA., \& Stern, PJ., (2003). Metacarpophalangeal joint arthroplasty in rheumatoid arthritis: A long-term assessment. The Journal of bone and joint surgery (American volume). 8-A5, 1869-1878.

Hume, MC., Gellman, H., McKellop, H., \& Brumfield, RH. Jr. (1990). Functional range of motion of the joints of the hand. The Journal of hand surgery. 15, 240-243. [Medline] [CrossRef]

Mannerfelt, L., \& Andersson, K. (1975). Silastic arthroplasty of the metacarpophalangeal joints in rheumatoid arthritis. Journal of Bone and Joint Surgery. 57, 484-489. [Medline]

Moller, K., Sollerman, C., Geijer, M., Kopyylov, P., \& Tagil, M. (2005). Avanta versus Swanson silicone implants in the MCP joint: A prospective, randomized comparison of 30 patients followed for 2 years. Journal of Hand Surgery. 30, 8-13. (British and European). [Medline]

Moran, SL., \& Berger, RA. (2003). Biomechanics and hand trauma: What you need. Hand clinics. 19, 17-31. [Medline] [CrossRef]

O'Brien, ET. (1996). Surgical principles and planning for the rheumatoid hand and wrist. Clinics in Plastic Surgery. 23, 407-420. [Medline]

O’Brien, AV., Jones, P., Mullis, R., Mulherin, D., \& Dziedzic, K. (2006). Conservative hand therapy treatments in rheumatoid arthritis: A randomized controlled trial. Rheumatology. 45, 577-583. [Medline] [CrossRef]

Rothwell, AG., Cragg, KJ., \& O’Neill, LB. (1997). Hand function following silastic arthroplasty of the metacarpophalangeal joints in the rheumatoid hand. Journal of Hand Surger (Edinburgh, Scotland)y. 22, 90-93. [Medline]

Swanson, AB. (1972). Flexible implant arthroplasty for arthritics finger joints: Rationale, technique, and results of treatment. Journal of Bone and Joint Surgery. 54, 435455. [Medline] 